\title{
TOWARDS SDG 4: TRADE-OFFS FOR GEOSPATIAL OPEN EDUCATIONAL RESOURCES
}

\author{
S. Coetzee ${ }^{1, *}$, V. Rautenbach ${ }^{1}$, A. Çöltekin ${ }^{2}$, C. Pettit ${ }^{3}$, M. Madden ${ }^{4}$, S. Christophe ${ }^{5}$ and O. Lkhamjav ${ }^{6}$ \\ ${ }^{1}$ Department of Geography, Geoinformatics and Meteorology, University of Pretoria, Pretoria, South Africa - (serena.coetzee, \\ victoria.rautenbach)@up.ac.za \\ ${ }^{2}$ Institute for Interactive Technologies, University of Applied Sciences and Arts Northwestern Switzerland, Switzerland - \\ arzu.coltekin@fhnw.ch \\ ${ }^{3}$ City Futures Research Centre, Faculty of Built Environment, UNSW - c.pettit@unsw.edu.au \\ ${ }^{4}$ Center for Geospatial Research Department of Geography University of Georgia Athens, Georgia, USA - mmadden@uga.edu \\ ${ }^{5}$ COGIT Lab, IGN-France, Paris, France - sidonie.christophe@ign.fr \\ ${ }^{6}$ Chair of the Mongolian Geospatial Association Ulaanbaatar, Mongolia - ochirkhuyag@geomedeelel.mn
}

KEY WORDS: geospatial, educational resource, open education, catalogue, learning object metadata, metadata, teaching

\begin{abstract}
:
Through the ISPRS scientific initiative presented in this paper, we aim to make geospatial educational resources available and discoverable to those who teach and those who want to learn. In earlier work, we designed and implemented a prototype catalogue for geospatial educational resources, aimed at a target audience in higher education. The success of search and discovery in any catalogue relies heavily on the metadata that describes catalogue entries. Initial feedback showed that users find it difficult to use some of the metadata elements in the prototype to describe their teaching materials. In order to better understand their difficulties and further refine the metadata for describing educational resources that are used for geospatial purposes specifically, we asked a number of participants to describe geospatial educational resources according to four sets of metadata attributes. This paper presents the results of the study and recommends a set of metadata attributes that are specifically useful for geospatial educational resources. Implementation trade-offs are discussed, e.g., deciding between metadata attributes that are very specific or more generic, and catalogue entries that are immediately available to Web search engines without any quality checks vs. catalogue entries that are moderated by a community of educators before publishing them. By providing metadata about geospatial educational resources, the international geospatial community can contribute to the UN Sustainable Development Goal 4 to ensure inclusive and equitable quality education and to promote lifelong learning opportunities for all.
\end{abstract}

\section{INTRODUCTION}

The term "Open Educational Resources" (OERs) was coined at a UNESCO Forum in 2002 to describe the (then emerging) global phenomenon of openly sharing educational resources. Technology-mediated approaches to deliver learning worldwide have accelerated this phenomenon. OERs can expand access to learning of better quality at lower cost (UNESCO, 2020). Learning materials that are freely available for adaptation and re-purposing support the UN Sustainable Development Goal 4 to ensure inclusive and equitable quality education and to promote lifelong learning opportunities for all. The OER Commons is a public digital library of OERs where teachers and lecturers can search and discover freely available educational materials (OER Commons, 2020). A search for 'geo' in the OER Commons returns only 22 results, yet, a large number of geospatial educational resources, such as tutorials and practical assignments, are available through various platforms, such as Wikis, GitHub, CKAN and Moodle. Through the ISPRS scientific initiative presented in this paper, we aim to make such 'hidden' geospatial educational resources available and discoverable to those who teach and those who want to learn. Furthermore, a well-moderated catalogue makes it possible to assess the quality of teaching materials before they are made public.

At the time of this writing, the current COVID-19 pandemic has emphasized the need for such a catalogue. The majority of student courses presented remotely during the COVID-19 pandemic were not designed for online teaching. They present face-to-face content remotely, as there was no time to redesign and develop them into proper online courses. As a result of this rush to move online, people are searching for educational resources that could make this move easier and they rely on search engines or social media, such as Twitter, where anyone can share their teaching materials. A well designed and moderated catalogue of geospatial educational resources could ensure that teaching material of appropriate quality finds its way into courses taught online. The pandemic has also revealed the importance of geographic information and the challenge to correctly present such information on maps. Journalists reporting about the pandemic need to understand how information was collected (e.g., location of deceased as an address of the hospital or the deceased's residential address), how this can be interpreted, how an individual's privacy can be protected and how information should be presented on maps (e.g., as relative values on choropleth maps). Several blogs commented on this matter (Field, 2020; Huffman, 2020), various code repositories were published (De Sabbata, 2020) and we anticipate that many more publications from GIScience and sister communities on this topic will follow. A catalogue that includes online training material for journalists would be useful.

\footnotetext{
* Corresponding author
} 
In earlier work, we designed and implemented a prototype catalogue for geospatial OERs, aimed at a target audience in higher education, such as universities, and members of the International Society for Photogrammetry and Remote Sensing (ISPRS) or GeoForAll. Requirements for the catalogue were collected by qualitatively reviewing our needs as educators. The prototype catalogue (https://isprs.education/) was developed using the latest web application technology. Once the basic functionalities were available, approximately 100 geospatial educational resources were identified and added to the catalogue (Rautenbach et al., 2019).

The success of search and discovery in any catalogue relies heavily on the metadata about the educational resources. After a review of potential metadata schemas, such as Dublin Core (ISO, 2009) and IEEE LOM (IEEE, 2002), we decided to use a subset of the IEEE LOM elements for the prototype catalogue. However, initial feedback showed that users find it difficult to use some of these elements to describe their teaching materials. We wanted to better understand their difficulties and further refine the metadata for describing educational resources that are used for geospatial purposes specifically.

In this paper, we present results of assessing the usability of the metadata in our prototype catalogue of geospatial OERs. The paper commences with a background section that briefly reviews metadata for geospatial educational resources and a brief description of the prototype. Next, we explain how metadata usability was assessed, followed by the presentation of the assessment results and a discussion of their implications for catalogue implementation trade-offs.

\section{BACKGROUND}

\subsection{Metadata}

Educational resources can only be 'discovered' and reused if there are accurate descriptions about them, i.e., metadata is required (Roy et al., 2010). Roy et al., (2020) reviewed several metadata standards for describing e-learning material, including Dublin Core (ISO 2009), the IEEE Learning Object Model (LOM) (IEEE, 2002) and metadata standards developed by the IMS Global Learning Consortium (http://www.imsglobal.org/), Advance Distributed Learning Initiative (http://www.adlnet.org) and CanCore Learning Resource Metadata Initiative (http://www.cancore.ca/). They found that most learning object repositories at the time implemented IEEE LOM.

Metadata provides a description of data or objects and is often maintained in catalogues where it can be searched and filtered. Over the years, several schemas have been proposed as blueprints for metadata. Such a schema specifies metadata elements and relationships between them. In the paragraphs that follow we briefly introduce the metadata schemas included in the usability assessment describe in this paper.

Dublin Core (ISO 2009) is a widely used general-purpose metadata schema than can be used for both digital and physical objects. It contains 15 well-defined elements for describing any digital or physical object, but it does not cater for the pedagogical aspects of educational resources (Roy et al. 2010). We included Dublin Core because it is widely used in all kinds of online catalogues.

The IEEE Learning Object Model (LOM) (IEEE, 2002) is a metadata schema developed for the purpose of describing objects used for learning, education or training. It comprises nine categories of metadata, and each category consists of several metadata elements that help to describe pedagogical aspects of the object, such as learning resource type, interactivity level and intended end user. The IEEE LOM schema is rather extensive, providing metadata elements to describe every possible aspect of a learning object, but sometimes a simpler schema is easier to maintain. In this study, we only included those IEEE LOM elements that were implemented in the prototype catalogue (Rautenbach et al., 2019)

ISO 19115:2003, Geographic information - Metadata, defines a schema for describing geographic information and services. It contains metadata elements to describe the "identification, the extent, the quality, the spatial and temporal schema, spatial reference, and distribution of digital geographic data" (ISO 19115:2003). We included ISO 19115:2003 in this study because of the lack of geospatially related metadata elements in the Dublin Core and IEEE LOM metadata schemas. The ISO 19115 standard is widely used in the geospatial community to describe geospatial data and services (Brodeur et al., 2019). For example, Research Data Australia (RDA https://researchdata.ands.org.au/) provides access to metadata for more than 140,000 data collections across various domains and the Australian Urban Research Infrastructure provides access to over 5,000 datasets described according to ISO 19115 metadata elements (Delaney and Pettit, 2014; Pettit et al., 2015; Pettit et al., 2020; Sinnott et al., 2014). One example of an AURIN data hub is CityData (https://citydata.be.unsw.edu.au/), comprising approximately 50 researcher-created datasets (as of 1st May 2020), an important digital asset to support city planning (Goodspeed et al., 2018; Leao et al., 2017; Pettit et al., 2016).

For this study, we also considered relevant metadata attributes that are used to describe open educational resources in the OER Commons (OER Commons, 2020). This platform can harvest metadata from other catalogues, e.g., metadata structured according to IEEE LOM or Dublin Core, and one can also add educational resources directly into the Commons.

\subsection{Prototype catalogue for geospatial OERs}

For the prototype, we reviewed the two well-known metadata schemas, Dublin Core and IEEE LOM, and decided to work with a subset of 20 of the 60 metadata elements from the IEEE LOM. We evaluated various existing applications, including Islandora, ePrints, AtoM, Zenodo and DSpace, for implementation of the catalogue. Although all of the evaluated applications had useful characteristics, two requirements were not fulfilled in any of these: the implementation of a profile of IEEE Standard for Learning Object Metadata (IEEE, 2002); and creating a metadata record without the need to upload a file. Strictly speaking, the majority of these applications are implementations for a repository (a place where things are deposited or stored) and not for implementing a catalogue (a list of descriptions of things). This motivated the design and development of a custom catalogue.

The prototype was built on Google's Firebase platform, a backend-as-a-service (BaaS). Firebase was selected as it would handle the data storage (as JSON objects), authentication (with OAuth2), and hosting of static files. An advantage of this configuration is also that our needs do not exceed what is offered on the free tier (Spark Plan) and this contributes to the long-term sustainability of the catalogue. 
The front end of the prototype was developed in the React JavaScript Framework. React was selected as it not only improves the development speed with reusable components but also improves the performance with optimized rendering algorithms. The JavaScript library for creating user interfaces allows the developer to break up the interface into components that can be updated without updating the entire page. Additionally, React also made it possible to create responsive interfaces that automatically adjust to the resolution of the device they are viewed on.

At the moment, the prototype is functional and allows any user to search for and view a resource in the catalogue. For the prototype, only users who sign-in with a Google account can add new resources. This is an interim security measure put in place to prevent bots from adding spam to the catalogue. In the final version of the catalogue we intend to add a user management feature where people can sign up with their institutional emails, or other email accounts of their choosing. In the prototype, all metadata attributes are required (mandatory) when adding a resource. The user is assisted with tooltips that provide hints, based on the IEEE LOM standard, to explain how some of the attributes should be populated.

In the prototype only the administrator can delete a resource. Eventually, this could be replaced with functionality where a user can request a resource to be deleted, accompanied with a justification for the request.

\section{METHOD}

To find out which metadata attributes (elements) are useful for describing and/or searching for educational resources, we asked each co-author to do the following:

1. Write down a description of a geospatial educational resource that you could use for one of your modules/courses.

2. Use one of the search engines (Google, Bing, etc.) to search for such a geospatial educational resource.

a. Write down the keywords that you used.

b. Once you have found a resource, record the URL and write down the information (concepts / descriptors / keywords) that made you decide whether to use it or not.

3. Add metadata according to Dublin Core, OER Commons, and IEEE LOM elements used in the prototype and ISO 19115 for two or three geospatial educational resources. For each, explain which attributes were useful, which were obsolete, and which were difficult to fill in.

Additionally, two postgraduate students also provided the above input. Based on the results, we recommend a set of metadata attributes suitable for geospatial educational resources and discuss how the results relate to trade-offs for catalogue implementation.

\section{RESULTS}

\subsection{Using search engines to find educational resources}

The kind of educational resources that participants searched for included tutorials, datasets, case studies, software and online courses. Most participants included a keyword related to education, e.g., 'training', 'tutorial', 'course' and 'teaching', when searching for an educational resource, but not all of them. As web searches go, the first list of results was not necessarily what participants were looking for, and they followed different strategies to iteratively refine the search until they found what they were looking for.

\subsection{Describing educational resources according to different metadata schemas}

In the following subsections, we explain how participants used the attributes of the different metadata schemas to describe their educational resources. The attribute name (in italics) is followed by its definition in brackets (where available), and after the colon, an assessment follows of how participants used the attribute to describe the resource. We also refer to their comments on whether attributes were useful, obsolete or difficult to fill in.

\subsubsection{Dublin Core:}

Contributor (entity responsible for making contributions to the resource): There was confusion between this attribute and the creator attribute. In most cases, participants assigned the same value to both.

Coverage (spatial or temporal topic of the resource, spatial applicability of the resource, or jurisdiction under which the resource is relevant): Participants used this attribute to specify a spatial coverage (e.g., 'Australia') or topics covered (e.g., 'spatial database management'), and in some cases, this attribute was irrelevant (e.g., for technical educational material on mobile data collection, where a specific area is not relevant).

Creator (entity primarily responsible for making the resource): As indicated above, this attribute was confused with the Contributor attribute.

Date (point or period of time associated with an event in the lifecycle of the resource): This definition is so wide that the attribute can be used to represent any date related to the educational resource, and this is what participants did. Values included the publication date and the temporal extent of the resource.

Description (account of the resource): All participants used this attribute to describe the educational resource. Some had longer descriptions, others shorter ones.

Format (file format, physical medium, or dimensions of the resource): All participants used this attribute to describe the format, e.g., 'wiki page', 'website' or 'PDF files'.

Identifier (unambiguous reference to the resource within a given context): Participants used this attribute for various purposes, e.g., for the URL, for a title or descriptor, or it was left empty.

Language (language of the resource): This attribute is rather simple to understand and was used by all participants.

Publisher (entity responsible for making the resource available): Only some participants provided the name of a publisher for the online educational resource. Some participants duplicated the value of the contributor and/or creator in this attribute. There seemed to be confusion on how to use it, however, in the case of an online textbook this attribute would be required, and it should therefore be included in an online catalogue. 
Relation (related resource): Most of the participants provided a URL of a related educational resource.

Rights (information about rights held in and over the resource): All participants used this attribute to state the name of the license under which the resource is available.

Source (related resource from which the described resource is derived): In most cases, this attribute was not used.

Subject (topic of the resource): Together with the Type and Description attributes, this attribute was used by all participants to help describe the purpose or intent of the educational resources, e.g., 'using spatial databases and SQL to analyse the data' and 'User studies in the field of geoinformatics'.

Title (name given to the resource): All participants used this attribute.

Type (nature or genre of the resource): Possible values for the 'Type' attribute in Dublin Core are Collection, Dataset, Event, Image, Interactive Resource, Moving Image, Physical Object, Service, Software, Sound Still Image, Text. Since participants were presented with a free format field, they used this attribute to describe the kind of resource, e.g., 'tutorial', 'lesson plan' or 'assignment'.

4.2.2 OER Commons: The following attributes are identical to the Dublin Core attributes: Description, Language, Subject, Title; and these attributes were used in the same way as corresponding attributes in Dublin Core: Authors, Media Format, License. They are therefore not further discussed here.

Provider (for content hosted on external sites) and Provider Set (set of resources provided by a specific provider): Participants did not seem to understand how to use these two attributes. Very often the value was a duplicate of Authors, or it was left empty.

Material Type (Activity/Lab, Assessment, Case Study, Dataset, Diagram/Illustration, Full Course, Game, Homework / Assignment, Interactive, Lecture, Lecture Notes, Lesson, Lesson Plan, Module, Primary Source, Reading, Simulation, Student Guide, Syllabus, Teaching/Learning Strategy, Textbook, Unit of Study. All participants used this attribute. While the Dublin Core metadata attribute is free text, in the OER Commons a list of possible values specific to educational material is provided.

Educational Level (Lower Primary, Upper Primary, Middle School, High School, Community College, College, Graduate / Professional, Career / Technical, Adult Education): All participants specified one or more targeted educational levels to describe their resource.

Primary User (Student, Teacher, Administrator, Parent, Librarian, Other): This attribute was used by all participants to describe the resource.

Educational Use (curriculum/instruction, assessment, professional development, informal education, other): This attribute was also used by most participants.

Subject Areas (Applied Science, Arts and Humanities, etc.): All participants used this attribute. However, in some cases more than one value was required to describe the resource, and the values ranged from very specific (e.g., user studies) to very broad (e.g., urban geography). Such information could be included equally well in a set of tags or keywords.

Tags: All participants used this attribute to describe the resource, similar to how one would use keywords.

Date Added: This date is typically added automatically when the resource is added to a catalogue.

4.2.3 IEEE LOM elements used in the prototype: The following attributes are identical to the Dublin Core attributes: Title, Description, Language; and these attributes were used in the same way as corresponding attributes in Dublin Core: Authors, Copyright. The following attributes were used similarly to how they were used in the same way as corresponding attributes in OER Commons: End User (Author, Learner, Manager, Teacher), Keywords. They are therefore not further discussed here.

Date: All participants used this attribute, some for a year, others for a specific date, or for an update cycle (e.g., 'monthly').

Date added or updated: All participants filled this attribute with the date of adding the information, and in the prototype the data is automatically added.

Duration: All participants used this attribute, although some could only estimate the duration.

Resource Type (Data, Diagram, Exam, Exercise, Experiment, Figure, Graph, Index, Lecture, Model, Narrative text, Problem statement, Questionnaire, Self assessment, Simulation, Slide, Software, Table). All participants used this attribute to describe their educational resources.

Semantic density (very low, low, medium, high, very high), Interactivity type (Active, Expositive, Mixed), Interactivity level (Low, Medium, High, Very High) and Difficulty (Very easy, Easy, Medium, Difficult, Very Difficult): All participants used these attributes, maybe only because possible values were provided, but many commented that it was difficult to decide on an appropriate value.

Context (Higher Education, School, Training, Other): All participants used this attribute.

Cost: Most resources were free, and if there was a cost, it was unknown. Since costs could change without informing catalogues with information about the resource, it might be better not to include this information in the metadata.

URL: Participants used this attribute, unless they described a resource that was not yet available online.

4.2.4 ISO 19115: The following attributes are identical to the Dublin Core attributes: Title, Language; and these attributes were used in the same way as corresponding attributes in Dublin Core: Abstract, Distribution Format, Responsible Part. These attributes were used similarly to how they were used in the same way as corresponding attributes in OER Commons: Metadata datestamp, and these similar to how they are used in the prototype: Online resource. They are therefore not further discussed here.

File Identifier (unique identifier for this metadata file): Some participants used this attribute in the same way as the URL attribute in other schemas, while quite a few did not use this 
attribute or filled it with a question mark, suggesting that there was some confusion about how to use it.

Reference Date (date for the cited resource): Participants used this date in different ways. Some recorded the date when the metadata was recorded, others may have used it as the publication date.

Geographic location (geographic area of the dataset): Similar to the Coverage attribute in Dublin Core, participants used this attribute to specify a spatial coverage (e.g., 'Australia') and in some cases did not assign a value because it was irrelevant (e.g., for technical educational material on mobile data collection, where a specific area is not relevant).

Character Set (full name of the character coding standard used for the metadata set): No participant used this attribute.

Topic Category (main theme(s) of the dataset): Some participants used this attribute in the same way as the Subject attribute in other schemas, while quite a few did not use this attribute, suggesting that there was some confusion about how to use it.

Quite a number of attributes from ISO 19115 turned out not to be useful for describing educational resources. These attributes are useful for geographic data, but probably too detailed to describe educational resources. Very few participants used these attributes, such as Spatial Resolution (factor which provides a general understanding of the density of spatial data in the dataset), Spatial Representation Type (method used to spatially represent geographic information), Reference System (description of the spatial and temporal reference systems used in the dataset), and Lineage (information about the events or source data used in constructing the data specified by the scope or lack of knowledge about lineage).

\subsection{Metadata attributes recommended for geospatial educational resources}

The results of section 4.2 helped us understand which attributes were found to be useful when describing an educational resource. Metadata attributes used by all participants in the same way seem to be unambiguous and relevant and we recommend that they be included. See Table 1 . We recommend including attributes that were deemed to be useful but used by participants in different ways. We propose to include them in a revised way in order to avoid any confusion and ambiguities. See Table 2. The other metadata attributes were found to be obsolete or did not add enough value to be included. The recommended attributes will facilitate searching (e.g., title, subject, description), filtering (e.g., date or language) and providing information that is useful for deciding whether to further investigate an item in the search results (e.g., the format or license for use).

\begin{tabular}{|l|l|l|l|l|}
\hline \multicolumn{1}{|c|}{ Recommended } & \multicolumn{1}{|c|}{ OER Commons } & \multicolumn{1}{c|}{$\begin{array}{c}\text { Prototype (based on } \\
\text { IEEE LOM) }\end{array}$} & \multicolumn{1}{c|}{ ISO 19115 } \\
\hline Title & Title & Title & Title & Title \\
Subject & Subject & Subject & - & Topic Category \\
Description & Description & Description & Description & Abstract \\
Format & Format & Media Format & - & Distribution Format \\
Language & Language & Language & Language & Language \\
Tags & - & Tags & Keywords & - \\
Author & Creator & Authors & Authors & Responsible Party \\
License for Use & Rights & License & Copyright & - \\
Publisher & Publisher & - & - & - \\
URL & - & - & URL & Online resource \\
\hline
\end{tabular}

Table 1. Recommended metadata attributes used by all participants in the same way

\begin{tabular}{|l|l|l|l|l|}
\hline \multicolumn{1}{|c|}{ Recommended } & \multicolumn{1}{|c|}{ Dublin Core } & \multicolumn{1}{|c|}{ OER Commons } & \multicolumn{1}{c|}{$\begin{array}{c}\text { Prototype (based on } \\
\text { IEEE LOM) }\end{array}$} & \multicolumn{1}{c|}{ ISO 19115 } \\
\hline Publication Date & Date & - & Date & Reference Date \\
Metadata Date & Date & Date Added & Date Added or Updated & Metadata Datestamp \\
Material Type & Type & Material Type & Resource Type & - \\
Target Audience & - & Educational Level, Primary User & Context, End Users & - \\
Educational Purpose & - & Educational Use & - & - \\
\hline
\end{tabular}

Table 2. Recommended metadata attributes revised for our purpose

We propose to resolve the confusion about how to use different date attributes by qualifying the date expected in the respective attributes in our prototype: 'Publication Date', i.e., the publication date of the educational material; and 'Metadata Date', i.e., the date on which the metadata was added or updated.

The 'Type' (Dublin Core), 'Material Type' (OER Commons) and 'Resource Type' (Prototype) can be used to describe the nature of the geospatial educational resource, each in a different way. The list of options for 'Material Type; in the OER Commons is the most comprehensive and best matched participants' expectations (all participants specified a value from the list). We therefore recommended including an attribute called 'Material Type' with the list of possible values provided in the OER Commons. If necessary, this list could be expanded in future. One could also consider adding more than one value per educational resource, as some educational resources may include multiple types of material, e.g., a set of assessments and homework/assignments.

The 'Educational Level' (OER Commons) and 'Context' (Prototype) attributes fulfil a very similar purpose. For 'Educational Level' a more extensive list of values is provided, 
however, some of these are very specific (e.g., Lower Primary, Upper Primary, Middle School) and may differ from one country to another. 'Primary User' (OER Commons) and 'End User' (Prototype) are also very similar to each other but could also be seen to overlap with 'Educational Level' and 'Context'. Neither of the lists include anyone beyond educational institutions, such as professionals or journalists, a significant omission. We therefore recommend including an attribute, 'Target Audience', with the following list of possible values: School Learners, University Students, Professionals, Other. Here one can also consider adding more than one value per educational resource, as the educational resources may be useful for more than one target audience.

Finally, we recommend that an optional free format text attribute, 'Additional Information', be included. This attribute can be used to add information that cannot be represented by the mandatory attributes.

\subsection{Implications for catalogue implementation trade-offs}

Metadata schemas are typically created for a specific purpose. With our recommended list of attributes, we are now adding to this plethora of metadata schemas. However, our results show that none of the current metadata schemas are suitable for our purpose. There are mechanisms (e.g., a crosswalk) for mapping and converting metadata between different schemas, however, the aggregation and disaggregation of metadata elements during this process can affect the usefulness of the resulting metadata.

In Section 4.3, we pointed out the pros and cons of the lists of values provided for different attributes. There is always a tradeoff between a long list of very specific values vs a shorter list of more general values. The results show that simplicity is an important usability feature for a catalogue about educational resources. The values also have to be applicable globally. Our choice of attributes and lists of values were guided by these observations.

Including a Digital Object Identifier (DOIs) as metadata attribute in the prototype catalogue can assist with making educational resources more discoverable through both general purpose and library search engines. However, our results show that a DOI is seldomly assigned to educational resources.

Even though there are commonalities between metadata schemas for educational resources and geospatial resources, the results show that educational resources cannot be described in the same way as geospatial data and services. Metadata about geospatial data requires very specific elements, for example the spatial resolution or lineage, which are essential for data but not relevant for an educational resource (unless it is a dataset). Also, many catalogues and portals for geospatial data already exist, and the aim with our scientific initiative is not to replace those catalogues but to link or integrate them. For example, one could include in the catalogue a single educational resource with metadata about a geoportal for geospatial datasets. Such an entry in the catalogue could help instructors to find real world datasets for use in their teaching material.

OER Commons focuses on open resources (limited to Public Domain and Creative Commons licenses), whereas our prototype catalogue aims at a wider variety of resources, including proprietary resources that are freely available. Apart from OER Commons, various other catalogues for open educational resources have been developed. See for example, $\mathrm{OERu} \quad$ (https://oeru.org/), OpenEdu (https://www.open.edu/openlearn/),

Curriki (https://www.curriki.org/), and ShareMyLesson (https://sharemylesson.com/). With so many catalogues around, instructors may ask themselves where they should add metadata about their teaching materials, or where to look for such material. Would it be possible to integrate them into a single platform? Harvesting metadata between different catalogues is one option.

We also asked ourselves whether a catalogue with a search and filter interface is really needed. Katumba and Coetzee (2017) showed that geospatial catalogue items published as HTML pages marked up with Schema.org and Dublin Core improved the visibility and facilitated the discovery of geospatial resources on the Web. Most people use search engines such as Google and Bing when they look for something on the Web. If there is more relevant information about a webpage, it is easier for search engines to understand what the page is about, and the page can be ranked more appropriately in search results (UK Geospatial Commission, 2020).

In other words, our prototype catalogue could be used to add, moderate and maintain metadata about geospatial educational resources, which could then be made available for harvesting by other catalogues. The catalogue information could also be made available in a format that web search engines can index. Both of these approaches would enhance discoverability of geospatial educational resources. The concern with search engines is that with the vast volumes of information on the Web, it is a challenge to identify educational resources of good quality.

Google's solution to the quality challenge for datasets returned in search engine results is Google Dataset Search (https://datasetsearch.research.google.com/). Datasets (e.g., a CSV file, images, data in proprietary format or even training parameters for machine learning) are indexed in Google Dataset Search if a metadata file (i.e., JSON-LD, RDFa or microdata) to describe the dataset is available on the Web. There are strict quality guidelines to be adhered to, otherwise the dataset may be marked as spam. A similar approach for educational resources would also solve the quality challenge. A catalogue that is moderated by a community of educators (e.g., members of the GeoForAll or the ISPRS Commission on Education) can overcome the quality challenge.

\section{CONCLUSION}

In this paper we reported the results of a study to assess the usability of metadata attributes in a prototype catalogue of geospatial educational resources. Based on how participants used metadata elements to describe geospatial educational resources, we recommend a set of metadata attributes that are useful for a catalogue of geospatial educational resources. These attributes will be implemented in the next version of our catalogue.

This paper focussed on the usability and understandability of metadata attributes. The overall usability of the catalogue has not yet been tested. However, we plan conducting workshops with potential users over the next year to assess its usability and to get feedback to improve the interface and interaction in a next phase of implementation. For example, how would an average user search for a specific resource, and how long would it take them to get to the information they need?

One lesson we learnt was that while machine learning may be useful for automatically classifying and generating metadata 
about educational resources, one would probably always need a human moderator to review and accept submissions to the catalogue to ensure that items are relevant and not spam.

The project and funding of the prototype catalogue have come to an end, and this paper has provided some suggestions for taking the initiative further. In an era of digital transformation and a world where traditional classroom-type educational models are challenged, there is a growing need for the development and cataloguing of freely available educational resources (Hylén, 2006; Littlejohn, 2003). We recommend that geospatial educational resources have appropriate metadata and are registered in catalogues to support discovery and re-use. The international geospatial community can promote inclusive and high-quality geospatial education by providing such metadata about geospatial OERs to address this growing need.

\section{ACKNOWLEDGEMENTS}

This research was supported by a grant from the 2018 ISPRS Education and Capacity Building Initiatives. We would also like to thank Azile Mdleleni and Cameron Green who described geospatial educational resources according to the metadata schemas.

\section{REFERENCES}

Brodeur, J., Coetzee, S,. Danko, D., Garcia, S., Hjelmager, J., 2019. Geographic Information Metadata-An Outlook from the International Standardization Perspective. ISPRS International Journal of Geo-Information, 8, 280.

Delaney, P., Pettit, C.J., 2014. Urban Data Hubs Supporting Smart Cities In: S. Winter and C. Rizos (Eds.): Research@Locate'14, Canberra, Australia, 7-9 April 2014, http://ceur-ws.org, pp13-25.

De Sabbata, S., 2020. covid-19-it-analysis. Github repository. https://github.com/sdesabbata/covid-19-it-analysis (May 2020).

Field, K., 2020. Mapping coronavirus, responsibly. https://www.esri.com/arcgis-

blog/products/product/mapping/mapping-coronavirusresponsibly/ (3 May 2020).

Goodspeed, R., Pelzer, P., Pettit, C., 2018. Planning our Future Cities: The Role Computer Technologies can Play. In: Urban Planning Knowledge and Research, edited by Sanchez, T.W., Routledge, pp210-225.

Huffman, D., 2020. How to map stuff: a live community sharing event, https://somethingaboutmaps.wordpress.com/2020/03/19/howto-do-map-stuff/ (3 May 2020).

Hylén, J, 2006. Open Educational Resources: Opportunities and Challenges, Proceedings of Open Education, 49-63.

IEEE 1484.12.1-2002, IEEE Standard for Learning Object Metadata, https://standards.ieee.org/standard/1484_12_12002.html (3 May 2020)

ISO 15836-1:2009, Information and documentation -- The Dublin Core metadata element set -- Part 1: Core elements, International Organization for Standardisation (ISO), Geneva, Switzerland.
Katumba, S., Coetzee, S., 2017. Employing Search Engine Optimization (SEO) Techniques for Improving the Discovery of Geospatial Resources on the Web. ISPRS International Journal of Geo-Information, 6(9), 284-304

Leao, S.Z., Lieske, S.N., Conrow, L., Doig, J., Mann, V., Pettit, C.J., 2017. Building a National-Longitudinal Geospatial Bicycling Data Collection from Crowdsourcing. Urban Science, 2017, 1 (3), 23; doi:10.3390/urbansci1030023.

Littlejohn, A., 2003. 'Issues in Reusing Online Resources'. In Reusing online resources: a sustainable approach to e-learning.

OER Commons (2020). Website, available online at https://www.oercommons.org/ (3 May 2020).

OER Commons (2020). Material Types. OER Commons Help Center.

https:/help.oercommons.org/support/solutions/articles/4200004 6908-material-types (3 May 2020).

Pettit, C.J., Lieske, S.N., and Leao, S.Z., 2016 Big Bicycle Data Processing: From Personal Data to Urban Applications, ISPRS Ann. Photogramm. Remote Sens. Spatial Inf. Sci., III-2, 173179, https://doi.org/10.5194/isprs-annals-III-2-173-2016.

Pettit, C., Stimson, B., Barton, J., Goldie, X., Greenwood, P., Lovelace, R., Eagleson, S., 2020. Open access, open source and cloud computing: a glimpse into the future of GIS. In: Handbook of Planning Support Science, edited by Geertman, S., Stillwell, J.. Edward Elgar Publishing. pp56-72.

Pettit, C.J., Barton, J., Goldie, X., Sinnott, R., Stimson, R., Kvan, T., 2015. The Australian Urban Intelligence Network supporting Smart Cities. In: Planning Support Systems and Smart Cities, Lecture Notes in Geoinformation and Cartography, edited by Geertman S., Stillwell J., Ferreira J. and Goodspeed J. (eds). Springer, pp 243-259.

Rautenbach, V., Coetzee, S., Çöltekin, A., Pettit, C., Pijper, L., Madden, M., Christophe, S., Lkhamjav, O., 2019. Towards establishing an open catalogue for geospatial educational resources, Int. Arch. Photogramm. Remote Sens. Spatial Inf. Sci., XLII-2/W13, 1609-1614, https://doi.org/10.5194/isprsarchives-XLII-2-W13-1609-2019.

Roy, D., Sarkar, S., Ghose, S., 2010. A Comparative Study of Learning Object Metadata, Learning Material Repositories, Metadata Annotation \& an Automatic Metadata Annotation Tool. Advances in Semantic Computing, 2, 103-120.

Sinnott, R.O., Bayliss, C., Bromage, C., Galang, G., Grazioli, P., Greenwood, P., Macauley, A., et al., 2014. The Australia Urban Research Gateway, Concurrency and Computation: Practice and Experience, 27(2), 358-375.

UK Geospatial Commission, 2020. Search engine optimisation for publishers: Best practice guide, https://www.gov.uk/government/publications/search-engineoptimisation-for-publishers-best-practice-guide (3 May 2020).

UNESCO (2020). Website, www.unesco.org (3 May 2020). 